\title{
List total weighting of graphs
}

\author{
Tsai-Lien Wong * Daqing Yang ${ }^{\dagger} \quad$ Xuding Zhu ${ }^{\ddagger}$
}

January 16, 2009

\begin{abstract}
A graph $G=(V, E)$ is $\left(k, k^{\prime}\right)$-total weight choosable if the following is true: For any $\left(k, k^{\prime}\right)$-total list assignment $L$ that assigns to each vertex $v$ a set $L(v)$ of $k$ real numbers as permissible weights, and assigns to each edge $e$ a set $L(e)$ of $k^{\prime}$ real numbers as permissible weights, there is a proper $L$-total weighting, i.e., a mapping $f: V \cup E \rightarrow \mathbb{R}$ such that $f(y) \in L(y)$ for each $y \in V \cup E$, and for any two adjacent vertices $u$ and $v, \sum_{e \in E(u)} f(e)+f(u) \neq \sum_{e \in E(v)} f(e)+f(v)$. This paper introduces a method, the max-min weighting method, for finding proper $L$-total weightings of graphs. Using this method, we prove that complete multipartite graphs of the form $K_{n, m, 1,1, \ldots, 1}$ are $(2,2)$-total weight choosable and complete bipartite graphs other than $K_{2}$ are $(1,2)$-total weight choosable.
\end{abstract}

Keywords: Total weighting, edge weighting, vertex colouring.

\section{Introduction}

Suppose $G=(V, E)$ is a graph. For a vertex $v$ of $G$, let $E(v)$ be the set of edges of $G$ incident to $v$, and let $E^{*}(v)=E(v) \cup\{v\}$. An edge weighting of $G$ is a mapping that assigns to each edge $e$ of $G$ a real number $f(e)$. An

\footnotetext{
${ }^{*}$ Department of Applied Mathematics, National Sun Yat-sen University. Grant number: NSC97-2115-M-110-004-MY2

${ }^{\dagger}$ Center for Discrete Mathematics, Fuzhou University, Fuzhou, Fujian 350002, China. Supported in part by NSFC under grant 10771035, SRF for ROCS of SEM and grant SX2006-42 of colleges of Fujian.

${ }^{\ddagger}$ Department of Applied Mathematics, National Sun Yat-sen University, Kaohsiung, Taiwan 80424, and National Center for Theoretical Sciences. Grant number: NSC972115-M-110-008-MY3
} 
edge weighting $f$ induces a vertex colouring $\varphi_{f}: V \rightarrow \mathbb{R}$ of $G$, defined as $\varphi_{f}(v)=\sum_{e \in E(v)} f(e)$. We say $f$ is a proper edge weighting if the induced vertex colouring $\varphi_{f}$ is proper, i.e., for any edge $u v$ of $G, \varphi_{f}(u) \neq \varphi_{f}(v)$. The study of edge weighting was initiated by Karoński, Łuczak and Thomason [9]. They made an interesting conjecture: Every connected graphs $G \neq K_{2}$ has a proper edge weighting $f$ such that $f(e) \in\{1,2,3\}$ for every edge $e$. This conjecture is still open. It was shown in [3] that the conjecture would be true if the set $\{1,2,3\}$ is replaced by $\{1,2, \ldots, 30\}$. The result was improved in [2], where it was shown that the conjecture would be true if the set is $\{1,2, \ldots, 16\}$, and recently, it is shown in [10] that the conjecture would hold if the set is $\{1,2,3,4,5\}$.

Bartnicki, Grytczuk and Niwczykthe [7] considered the choosability version of edge weighting. A graph is said to be $k$-edge weight choosable if the following is true: For any list assignment $L$ which assigns to each edge $e$ a set $L(e)$ of $k$ real numbers, $G$ has a proper edge weighting $f$ such that $f(e) \in L(e)$ for each edge $e$. They conjectured that every graph without isolated edges is 3-edge weight choosable, and verified the conjecture for complete graphs, complete bipartite graphs and some other graphs.

A total weighting of $G$ is a mapping $f: V \cup E \rightarrow \mathbb{R}$ which assigns to each vertex and each edge a real number as its weight. For a total weighting $f$, let $\varphi_{f}: V \rightarrow \mathbb{R}$ be defined as $\varphi_{f}(v)=\sum_{y \in E^{*}(v)} f(y)$. A total weighting is proper if the induced vertex colouring $\varphi_{f}$ is proper. Przybyło and Woźniak $[11,12]$ studied total weighting of graphs. They conjectured that every simple graph $G$ has a proper total weighting $f$ such that $f(y) \in\{1,2\}$ for all $y \in V \cup E$, and verified this conjecture for some special graphs, including complete graphs, 4-regular graphs and graphs $G$ with $\chi(G) \leq 3$. They also proved that every simple graph $G$ has a proper total weighting $f$ such that $f(y) \in\{1,2, \ldots, 11\}$ for all $y \in V \cup E$. This result was improved in [10] where it was shown $f$ can be chosen so that $f(v) \in\{1,2\}$ for every vertex $v$ and $f(e) \in\{1,2,3\}$ for every edge $e$.

The choosability version of total weighting was studied in [13]. For positive integers $k, k^{\prime}$, a $\left(k, k^{\prime}\right)$-total list assignment is a mapping $L$ which assigns to each vertex $v$ a set $L(v)$ of $k$ real numbers as permissible weights, and assigns to each edge $e$ a set $L(e)$ is $k^{\prime}$ real numbers as permissible weights. A graph is called $\left(k, k^{\prime}\right)$-total weight choosable if for any $\left(k, k^{\prime}\right)$-total list assignment $L, G$ has a proper total weighting $f$ such that $f(y) \in L(y)$ 
for all $y \in V \cup E$. In the following, we say a graph is $\left(k, k^{\prime}\right)$-choosable if it is $\left(k, k^{\prime}\right)$-total weight choosable. It is known [13] that a graph is $(k, 1)$ choosable if and only if it is $k$-choosable. So the concept of $\left(k, k^{\prime}\right)$-choosable builds a bridge between the concept of vertex colouring and edge weighting. The following conjectures were proposed in [13]:

Conjecture 1.1. Every graph is (2,2)-choosable.

Conjecture 1.2. Every graph with no isolated edges is $(1,3)$-choosable.

However, it is still unknown if there are constants $k, k^{\prime}$ such that every graph is $\left(k, k^{\prime}\right)$-choosable. It was shown in [13] that complete graphs, trees, cycles, generalized theta graphs are $(2,2)$-choosable, and complete bipartite graphs $K_{2, n}$ are $(1,2)$-choosable and $K_{3, n}$ are $(2,2)$-choosable.

The method used in [7] and in [13] for the study of edge weight choosability or total weight choosability of graphs is algebraic (see Section 5), which proves the existence of a proper edge weighting or proper total weighting without actually constructing such a weighting.

In this paper, we introduce a method that constructs explicitly a proper total weighting of given graphs. Then we apply this method and prove that complete multipartite graphs of the form $K_{n, m, 1,1, \ldots, 1}$ are $(2,2)$-choosable and complete bipartite graphs other than $K_{2}$ are $(1,2)$-choosable.

\section{The max-min weighting method}

Suppose $L$ is a $\left(k, k^{\prime}\right)$-total list assignment of a graph $G$. The max-min weighting method finds a proper $L$-weighting of $G$ step by step. At each step, we find a vertex $v$ and determine the weights of all the elements of $E^{*}(v)$. Let $f_{0}: E^{*}(v) \rightarrow \mathbb{R}$ be a map such that for each $y \in E^{*}(v), f_{0}(y) \in L(y)$. We call $f_{0}$ a partial $L$-weighting of $G$ for vertex $v$. If $f_{0}$ is a partial $L$-weighting of $G$ for $v$, let $\varphi_{f_{0}}(v)=\sum_{y \in E^{*}(v)} f_{0}(y)$. Then for any total weighting $f$ of $G$ which coincides with $f_{0}$ on $E^{*}(v)$, we have $\varphi_{f}(v)=\varphi_{f_{0}}(v)$. I.e., the colour of $v$ is determined by the partial $L$-weighting $f_{0}$.

Given a partial $L$-weighting $f_{0}$ of $G$ for vertex $v$. We define a $\left(k, k^{\prime}\right)$-total list assignment $L_{1}$ for $G_{1}=G-v$ as follows:

$L_{1}(y)=\left\{\begin{array}{l}L(y), \text { If } y \text { is a vertex not adjacent to } v \text { or } y \text { is an edge of } G_{1}, \\ \left\{w+f_{0}(e): w \in L(y)\right\}, \text { If } y \text { is a vertex adjacent to } v \text { and } e=y v .\end{array}\right.$ 
We call $L_{1}$ the $\left(k, k^{\prime}\right)$-total list assignment induced by $L$ and $f_{0}$.

Suppose $L_{1}$ is the $\left(k, k^{\prime}\right)$-total list assignment of $G_{1}$ induced by $L$ and $f_{0}$. If $f_{1}$ is an $L_{1}$-weighting of $G_{1}$, then we combine $f_{1}$ and $f_{0}$ to obtain a weighting $f$ of $G$ as follows:

$$
f(y)=\left\{\begin{array}{l}
f_{1}(y)-f_{0}(e), \text { If } y \text { is a vertex adjacent to } v \text { and } e=y v, \\
f_{1}(y), \text { If } y \text { is a vertex not adjacent to } v \text { or } y \text { is an edge of } G_{1}, \\
f_{0}(y), \text { If } y \in E^{*}(v)
\end{array}\right.
$$

We call $f$ the $L$-weighting of $G$ induced by $f_{1}$ and $f_{0}$. The following lemma is obvious and its proof is omitted.

Lemma 2.1. Suppose $L$ is a $\left(k, k^{\prime}\right)$-total list assignment of a graph $G, f_{0}$ is a partial $L$-weighting of $G$ for vertex $v$, and $L_{1}$ is the $\left(k, k^{\prime}\right)$-total list assignment of $G_{1}=G-v$ induced by $L$ and $f_{0}$. If $f_{1}$ is an $L_{1}$-weighting of $G_{1}$, and $f$ is the $L$-weighting of $G$ induced by $f_{1}$ and $f_{0}$, then for each $u \in V\left(G_{1}\right), \varphi_{f}(u)=\varphi_{f_{1}}(u)$ and $\varphi_{f}(v)=\varphi_{f_{0}}(v)$.

For each $y \in V \cup E$, let $L_{\max }(y)=\max L(y)$ be the maximum number in $L(y)$, and let $L_{\min }(y)=\min L(y)$ be the minimum number in $L(y)$. Note that if $|L(y)|=1$, then $L_{\max }(y)=L_{\min }(y)$. Otherwise, $L_{\max }(y)>L_{\min }(y)$.

Let

$$
\begin{aligned}
\varphi_{L, \max }(v) & =\sum_{y \in E^{*}(v)} L_{\max }(y) \\
\varphi_{L, \min }(v) & =\sum_{y \in E^{*}(v)} L_{\min }(y) .
\end{aligned}
$$

It follows from the definition that $\varphi_{L, \max }(v)=\max \varphi_{f}(v)$ and $\varphi_{L, \min }(v)=$ $\min \varphi_{f}(v)$, where the maximum and minimum is taken over all $L$-weightings $f$ of $G$. So for any $L$-weighting $f$ of $G$, we have

$$
\varphi_{L, \min }(v) \leq \varphi_{f}(v) \leq \varphi_{L, \max }(v)
$$

For a vertex $v$ of $G, N_{G}(v)=\{u: u$ is a neighbour of $v\}$, and $N_{G}[v]=$ $N_{G}(v) \cup\{v\}$. A vertex $v$ of $G$ is locally maximum (with respect to $L$ ) if for any $u \in N_{G}(v), \varphi_{L, \max }(u) \leq \varphi_{L, \max }(v)$, and $v$ is locally minimum (with respect to $L)$ if for any $u \in N_{G}(v), \varphi_{L, \min }(u) \geq \varphi_{L, \min }(v)$.

A partial $L$-weighting of $G$ for a vertex $v$ is said to max-weights $v$ if $f(y)=L_{\max }(y)$ for each $y \in E^{*}(v)$. A partial $L$-weighting of $G$ for a vertex 
$v$ is said to min-weights $v$ if $f(y)=L_{\min }(y)$ to for each $y \in E^{*}(v)$. If $f$ is a partial $L$-weighting of $G$ that max-weights $v$, then $\varphi_{f}(v)=\varphi_{L, \max }(v)$; if $f$ is a partial weighting of $G$ that min-weights $v$, then $\varphi_{f}(v)=\varphi_{L, \min }(v)$.

Suppose we have defined a partial $L$-weighting $f_{0}$ of $G$ for vertex $v$. Let $L_{1}$ be the $\left(k, k^{\prime}\right)$-total list assignment induced by $L$ and $f_{0}$. By Lemma 2.1, to find a proper $L$-weighting of $G$, it suffices to find a proper $L_{1}$-weighting $f_{1}$ of $G_{1}$ so that for each $u \in N_{G}(v), \varphi_{f_{1}}(u) \neq \varphi_{f_{0}}(v)$.

Our max-min-weighting method will only max-weight locally maximum vertices, and min-weight locally minimum vertices. By doing so, the conflicts with vertices "coloured" in later steps will not be too complicated. Indeed, assume $v$ is a locally maximum vertex, and $f_{0}$ is a partial $L$-weighting of $G$ for $v$ that max-weights $v$. Let

$$
Q_{L, \max }(v)=\left\{u \in N_{G}(v): \varphi_{L, \max }(u)=\varphi_{L, \max }(v)\right\}
$$

To extend $f_{0}$ to a proper $L$-weighting $f$ of $G$, we need to make sure that no vertex $u \in Q_{L, \max }(v)$ will be "coloured" with $\varphi_{L, \max }(u)$. For this purpose, it suffices that some $y \in E^{*}(u)$ is assigned a weight smaller than $L_{\max }(y)$. I.e., $u$ is not max-weighted (under the list $L$ ). Similarly, assume $v$ is a locally minimum vertex, and $f_{0}$ is a partial $L$-weighting of $G$ for $v$ that min-weights $v$, and

$$
Q_{L, \min }(v)=\left\{u \in N_{G}(v): \varphi_{L, \min }(u)=\varphi_{L, \min }(v)\right\} .
$$

To extends $f_{0}$ to a proper $L$-weighting $f$ of $G$, we need to make sure that $u \in Q_{L, \min }(v)$ is not min-weighted (under the list $L$ ). This motivates the following definition.

Definition 2.2. Suppose $G=(V, E)$ is a graph and $L$ is a $\left(k, k^{\prime}\right)$-total list assignment of $G$. Let $S_{0}, T_{0}$ be two subsets of $V$ (each of $S_{0}, T_{0}$ may be empty and $S_{0}, T_{0}$ need not be disjoint). A proper $\left(L ; S_{0}, T_{0}\right)$-weighting of $G$ is a proper $L$-weighting $f$ of $G$ such that for each $v \in S_{0}, \varphi_{f}(v) \neq \varphi_{L, \max }(v)$ and for each $v \in T_{0}, \varphi_{f}(v) \neq \varphi_{L, \min }(v)$.

The following theorem contains the key idea of max-min weighting method.

Theorem 2.3. Suppose $G=(V, E)$ is a graph, $k^{\prime} \geq 2$ and $L$ is a $\left(k, k^{\prime}\right)$-total list assignment of $G$, and $S_{0}, T_{0}$ are two subsets of $V$.

- Assume $v$ is a locally maximum vertex and $v \notin S_{0}$. In case $k=1$, we further assume that $v$ is not an isolated vertex. Let $f_{0}$ be the partial 
$L$-weighting of $G$ that max-weights $v$. Let $G_{1}=G-v$, and let $L_{1}$ be the $\left(k, k^{\prime}\right)$-total list assignment induced by $L$ and $f_{0}$. Let

$$
S_{1}=S_{0} \cup Q_{L, \max }(v), \quad T_{1}=T_{0}-N_{G}[v] .
$$

If $f_{1}$ is a proper $\left(L_{1} ; S_{1}, T_{1}\right)$-weighting of $G_{1}$, then the L-weighting induced by $f_{1}$ and $f_{0}$ is a proper $\left(L ; S_{0}, T_{0}\right)$-weighting of $G$.

- Assume $v$ is a locally minimum vertex and $v \notin T_{0}$. In case $k=1$, we further assume that $v$ is not an isolated vertex. Let $f_{0}$ be the partial $L$-weighting of $G$ that min-weights $v$. Let $G_{1}=G-v$, and let $L_{1}$ be the $\left(k, k^{\prime}\right)$-total list assignment induced by $L$ and $f_{0}$. Let

$$
S_{1}=S_{0}-N_{G}[v], \quad T_{1}=T_{0} \cup Q_{L, \min }(v) .
$$

If $f_{1}$ is a proper $\left(L_{1} ; S_{1}, T_{1}\right)$-weighting of $G_{1}$, then the L-weighting induced by $f_{1}$ and $f_{0}$ is a proper $\left(L ; S_{0}, T_{0}\right)$-weighting of $G$.

Proof. Assume $v$ is a locally maximum vertex and $v \notin S_{0}$. Let $f_{0}$ be the partial $L$-weighting of $G$ that max-weights $v$. Let $G_{1}, S_{1}, T_{1}$ be defined as above. Assume $f_{1}$ is a proper $\left(L_{1} ; S_{1}, T_{1}\right)$-weighting of $G_{1}$. Let $g$ be the $L$-weighting of $G$ induced by $f_{1}$ and $f_{0}$. To prove that $g$ is a proper ( $L ; S_{0}, T_{0}$ )-weighting of $G$, it suffices to show the following:

1. For any $u \in Q_{L, \max }(v), \varphi_{g}(u) \neq \varphi_{L, \max }(u)$.

2. For any $u \in S_{0}, \varphi_{g}(u) \neq \varphi_{L, \max }(u)$.

3. For any $u \in T_{0}, \varphi_{g}(u) \neq \varphi_{L, \min }(u)$.

By Lemma 2.1, $\varphi_{g}(u)=\varphi_{f_{1}}(u)$ for $u \neq v$ and $\varphi_{g}(v)=\varphi_{L, \max }(v)$. Since $Q_{L, \max }(v) \subseteq S_{1}$ and $f_{1}$ is a proper $\left(L_{1} ; S_{1}, T_{1}\right)$-weighting of $G$, it follows that for $u \in Q_{L, \text { max }}(v) \subseteq S_{1}, \varphi_{g}(u)=\varphi_{f_{1}}(u) \neq \varphi_{L, \text { max }}(u)$. So (1) is satisfied. Since $S_{0} \subseteq S_{1}$, we also have $\varphi_{g}(u)=\varphi_{f_{1}}(u) \neq \varphi_{L, \max }(u)$ for every $u \in S_{0}$. So (2) is satisfied. Assume $u \in T_{0}$. If $u \in T_{1}$, then $\varphi_{g}(u)=$ $\varphi_{f_{1}}(u)>\varphi_{L_{1}, \min }(u)=\varphi_{L, \min }(u)$. If $u \in N_{G}(v)$, then since the edge $e=u v$ is assigned the weight $g(e)=f_{0}(e)=L_{\max }(e)>L_{\min }(e)$ (here we use the assumption that $k^{\prime} \geq 2$ ), we conclude that $\varphi_{L_{1}, \min }(u)>\varphi_{L, \min }(u)$. Therefore $\varphi_{g}(u) \geq \varphi_{L_{1}, \min }(u)>\varphi_{L, \min }(u)$. If $u=v$, then since either $k \geq 2$ or $v$ is not isolated, we have $\varphi_{L, \max }(v)>\varphi_{L, \min }(v)$ (here again we use the 
assumption that $\left.k^{\prime} \geq 2\right)$. Therefore $\varphi_{g}(v)=\varphi_{L, \max }(v) \neq \varphi_{L, \min }(v)$. Thus (3) is satisfied.

The case that $v$ is locally minimum is symmetric, and the proof is the same, except that max is replaced by $\min , Q_{L, \max }(v)$ is replaced by $Q_{L, \min }(v)$, and $S_{0}, T_{0}$ are interchanged, and $S_{1}, T_{1}$ are interchanged.

\section{Application of the max-min method}

In this section, we use the max-min weighting method to prove that complete multipartite graphs of the form $K_{n, m, 1,1, \ldots, 1}$ are $(2,2)$-choosable and complete bipartite graphs other than $K_{2}$ are $(1,2)$-choosable.

In most of the application of the max-min weighting method in this paper, we max-weight globally maximum vertices and min-weight globally minimum vertices. For a $\left(k, k^{\prime}\right)$-total list assignment $L$ of $G$, let $V_{L, \max }(G)$ be the set of globally maximum vertices of $G$ and let $V_{L, \min }(G)$ be the set of globally minimum vertices of $G$. To be precise,

$$
\begin{aligned}
& V_{L, \max }(G)=\left\{v \in V(G): \forall u \in V(G), \varphi_{L, \max }(v) \geq \varphi_{L, \max }(u)\right\} \\
& V_{L, \min }(G)=\left\{v \in V(G): \forall u \in V(G), \varphi_{L, \min }(v) \leq \varphi_{L, \min }(u)\right\} .
\end{aligned}
$$

First we prove that complete multipartite graphs of the form $K_{n, m, 1,1, \ldots, 1}$ are $(2,2)$-choosable.

Theorem 3.1. Suppose $n, m, k \geq 0$ are integers. Let $G=K_{n, m, 1,1, \ldots, 1}$ be the complete multipartite graph with one partite set of size $n$, one partite set of size $m$ and $k$ partite sets of size 1 . Denote by $A$ the partite set of size $n$, and by $B$ the partite set of size $m$ and by $C$ the union of the $k$ partite sets each of size 1. Suppose $L$ is a $(2,2)$-total list assignment $L$ of $G$, and $S_{0}, T_{0}$ are subsets of $V(G)$ such that one of $S_{0}, T_{0}$ is empty and the other has an empty intersection with $A$ or $B$. Then there is a proper $\left(L ; S_{0}, T_{0}\right)$-weighting of $G$. Hence $G$ is $(2,2)$-choosable.

Proof. We prove this theorem by induction on $|V(G)|$. If $|V(G)|=1$, then the conclusion is obviously true. Assume $|V(G)|>1$. Without loss of generality, we assume that $S_{0}=\emptyset$ and assume that $T_{0} \cap A=\emptyset$.

- If $V_{L, \max }(G) \cap A \neq \emptyset$, then let $v \in V_{L, \max }(G) \cap A$.

- If $V_{L, \max }(G) \cap A=\emptyset$ and $V_{L, \max }(G) \cap C \neq \emptyset$, then let $v \in V_{L, \max }(G) \cap C$. 
- If $V_{L, \max }(G) \cap(A \cup C)=\emptyset$, then let $v \in V_{L, \max }(G) \cap B$.

Let $f_{0}$ be the partial $L$-weighting of $G$ that max-weights $v$. Let $G^{\prime}=G-v$ and $L_{1}$ be the $(2,2)$-total list assignment induced by $L$ and $f_{0}$. Let $S_{1}=$ $S_{0} \cup Q_{L, \max }(v)=Q_{L, \max }(v)$ and let $T_{1}=T_{0}-N_{G}[v]$. It is easy to verify that if $v \in A$, then $T_{1}=\emptyset$ and $S_{1} \cap A=\emptyset$. If $v \in C$, then $T_{1}=\emptyset$ and $S_{1} \cap A=\emptyset$. If $v \in B$, then $S_{1}=\emptyset$ and $T_{1} \cap A=\emptyset$. By induction hypothesis, $G^{\prime}$ has a proper $\left(L_{1} ; S_{1}, T_{1}\right)$-weighting. By Theorem 2.3, $G$ has a proper $\left(L ; S_{0}, T_{0}\right)$-weighting.

It was proved in [13] that complete graphs are $(2,2)$-choosable. The proof in [13] uses Combinatorial Nullstellensatz and does not provide an algorithm that constructs a proper $L$-total weighting of $K_{n}$ for a given $(2,2)$-total list assignment $L$ of $K_{n}$. The proof above gives a linear time algorithm that constructs a required proper $L$-total weighting of $K_{n, m, 1,1, \ldots, 1}$.

In [13], the problem whether complete bipartite graphs are $(2,2)$-choosable was studied. It was proved there that for any positive integer $n, K_{2, n}$ are $(1,2)$-choosable and $K_{3, n}$ are $(2,2)$-choosable. But the question whether all complete bipartite graphs are $(2,2)$-choosable remained open. Theorem 3.1 answers this question in the affirmative. Theorem 3.2 below shows that complete bipartite graphs are actually $(1,2)$-choosable, provided that it is not $K_{2}$.

Theorem 3.2. Suppose $G=K_{n, m} \neq K_{2}$ is a complete bipartite graph and $L$ is a (1,2)-total list assignment of $G$. If both $S_{0}, T_{0}$ are empty sets, then $G$ has a proper $\left(L ; S_{0}, T_{0}\right)$-weighting.

Proof. Assume the theorem is not true and $G=K_{n, m}$ is a counterexample with minimum number of vertices. Let $A, B$ be the two partite sets with $|A|=n$ and $|B|=m$.

Claim 1. $G \notin\left\{K_{1,2}, K_{1,3}, K_{2,2}, K_{2,3}\right\}$.

The graphs excluded in this claim are small graphs. One way to prove this claim is to do a case by case check, which seems to be tedious. Another method is to use Combinatorial Nullstellensatz, which will be discussed in Section 5 .

Claim 2. None of $V_{L, \max }(G)$ and $V_{L, \min }(G)$ is an independent set of $G$. 
Proof. Assume to the contrary that $V_{L, \max }(G)$ is an independent set of $G$, i.e., $V_{L, \max }(G)$ is a subset of $A$.

Let $v \in V_{L, \max }(G)$ and let $f_{0}$ be the partial $L$-weighting of $G$ that maxweights $v$. Let $L_{1}$ be the $(1,2)$-total list assignment of $G_{1}=G-v$ induced by $L$ and $f_{0}$. Since $S_{0}=T_{0}=\emptyset$, and $Q_{L, \max }(v)=\emptyset$, we have $S_{1}=T_{1}=\emptyset$ for the sets $S_{1}, T_{1}$ defined in Theorem 2.3. Since $G_{1} \neq K_{2}$, by induction hypothesis, $G_{1}$ has a proper $\left(L_{1} ; S_{1}, T_{1}\right)$-weighting $f_{1}$. By Theorem 2.3 , the $L$-weighting $f$ of $G$ induced by $f_{1}$ and $f_{0}$ is a proper $L$-weighting of $G$.

Let

$$
\begin{aligned}
A_{\max } & =V_{L, \max }(G) \cap A, \\
B_{\max } & =V_{L, \max }(G) \cap B, \\
A_{\min } & =V_{L, \min }(G) \cap A, \\
B_{\min } & =V_{L, \min }(G) \cap B .
\end{aligned}
$$

By Claim 2, none of the sets $A_{\max }, B_{\max }, A_{\min }, B_{\min }$ is empty.

Claim 3. $\left|A_{\max } \cup A_{\min }\right|=\left|B_{\max } \cup B_{\min }\right|=1$.

Proof. Assume to the contrary that $\left|A_{\max } \cup A_{\min }\right|>1$. Then there exist $v \in A_{\max }$ and $v^{\prime} \in A_{\min }$ such that $v \neq v^{\prime}$.

Let $f_{0}$ be the partial $L$-weighting of $G$ that max-weights $v$, and let $L_{1}$ be the (1,2)-total weighting of $G_{1}=G-v$ induced by $L$ and $f_{0}$. Then for any vertex $x \in B$,

$$
\varphi_{L_{1}, \min }(x)>\varphi_{L, \min }(x) \geq \varphi_{L, \min }\left(v^{\prime}\right)=\varphi_{L_{1}, \min }\left(v^{\prime}\right) .
$$

Therefore $v^{\prime} \in V_{L_{1}, \min }\left(G_{1}\right)$ and $V_{L_{1}, \min }\left(G_{1}\right) \cap B=\emptyset$.

Let $S_{1}=S_{0} \cup Q_{L, \max }(v)$ and $T_{1}=T_{0}-N_{G}[v]$. We have $T_{1}=\emptyset$ and $S_{1}=B_{\max }$.

Let $f_{1}$ be the partial $L_{1}$-weighting of $G_{1}$ that min-weights $v^{\prime}$ and let $L_{2}$ be the (1,2)-total list assignment of $G_{2}=G_{1}-v^{\prime}$ induced by $L_{1}$ and $f_{1}$. Let $S_{2}=S_{1}-N_{G_{1}}\left[v^{\prime}\right]$ and $T_{2}=T_{1} \cup Q_{L_{1}, \min }\left(v^{\prime}\right)$. It is obvious that $S_{2}=\emptyset$. Since $V_{L_{1}, \min }\left(G_{1}\right) \cap B=\emptyset$, we have $T_{2}=Q_{L_{1}, \min }\left(v^{\prime}\right)=\emptyset$. By the minimality of $G$, we know that $G_{2}$ has a proper $\left(L_{2} ; S_{2}, T_{2}\right)$-weighting. By Theorem 2.3, $G_{1}$ has a proper $\left(L_{1} ; S_{1}, T_{1}\right)$-weighting and $G$ has a proper $\left(L ; S_{0}, T_{0}\right)$-weighting. 
Assume $A_{\max }=A_{\min }=\{v\}$ and $B_{\max }=B_{\min }=\{u\}$.

Let $f_{0}$ be the partial $L$-weighting of $G$ that max-weights $v$, and let $L_{1}$ be the (1,2)-total list assignment of $G_{1}=G-v$ induced by $L$ and $f_{0}$. Then $S_{1}=S_{0} \cup Q_{L, \max }(v)=\{u\}$ and $T_{1}=T_{0}-N_{G}[v]=\emptyset$. By Theorem 2.3, if $G_{1}$ has a proper $\left(L_{1} ; S_{1}, T_{1}\right)$-weighting, then $G$ has a proper $\left(L ; S_{0}, T_{0}\right)$ weighting. As $S_{1} \neq \emptyset$, we cannot conclude (from the minimality of $G$ ) that $G_{1}$ has a proper $\left(L_{1} ; S_{1}, T_{1}\right)$-weighting.

If $V_{L_{1}, \min }\left(G_{1}\right) \subseteq A$, then let $v^{\prime} \in V_{L_{1}, \min }\left(G_{1}\right)$. Let $f_{1}$ be the partial $L_{1}$-weighting that min-weights $v^{\prime}$. Let $L_{2}$ be the $(1,2)$-total list assignment of $G_{2}=G_{1}-v^{\prime}$ induced by $L_{1}$ and $f_{1}$. Let $S_{2}=S_{1}-N_{G}\left[v^{\prime}\right]$ and $T_{2}=$ $T_{1} \cup Q_{L_{1}, \min }\left(v^{\prime}\right)$. Then $S_{2}=T_{2}=\emptyset$. Since $G_{2} \neq K_{2}$, by the minimality of $G$, $G_{2}$ has a proper $\left(L_{2} ; S_{2}, T_{2}\right)$-weighting. By Theorem 2.3 , this implies that $G_{1}$ has a proper $\left(L_{1} ; S_{1}, T_{1}\right)$-weighting, and hence $G$ has a proper $\left(L ; S_{0}, T_{0}\right)$ weighting.

Thus we may assume that $V_{L_{1}, \min }\left(G_{1}\right) \cap B \neq \emptyset$. Let

$$
B^{\prime}=V_{L_{1}, \min }\left(G_{1}\right) \cap B .
$$

By definition, for each $x \in B^{\prime}$, for each $y \in A-\{v\}, \varphi_{L_{1}, \min }(x) \leq \varphi_{L_{1}, \min }(y)$. However, it follows from the definition that $\varphi_{L_{1}, \min }(x)>\varphi_{L, \min }(x)$ and $\varphi_{L_{1}, \min }(y)=\varphi_{L, \min }(y)$. So the following claim holds.

Claim 4. For each $x \in B^{\prime}$ and for each $y \in A-\{v\}, \varphi_{L, \min }(x)<\varphi_{L, \min }(y)$.

Interchange the roles of $A$ and $B$, and let $f_{0}^{\prime}$ be the partial $L$-weighting of $G$ that max-weights $u$, let $L_{1}^{\prime}$ be the $(1,2)$-weighting of $G_{1}^{\prime}=G-u$ induced by $L$ and $f_{0}^{\prime}$, and let

$$
A^{\prime}=V_{L_{1}^{\prime}, \min }\left(G_{1}^{\prime}\right) \cap A .
$$

By symmetry, we have $A^{\prime} \neq \emptyset$ and the following claim holds.

Claim 5. For each $y \in A^{\prime}$ and for each $x \in B-\{u\}, \varphi_{L, \min }(y)<\varphi_{L, \min }(x)$.

By combining Claims 4 and 5, we can prove the following claim.

Claim 6. $A^{\prime}=\{v\}$ and $B^{\prime}=\{u\}$.

Proof. If $A^{\prime}-\{v\} \neq \emptyset$ and $B^{\prime}-\{u\} \neq \emptyset$, then for $y \in A^{\prime}-\{v\}$ and $x \in B^{\prime}-\{u\}$, Claims 4 and 5 contradicts each other. Thus, by symmetry, we may assume that $B^{\prime}-\{u\}=\emptyset$ and hence $B^{\prime}=\{u\}\left(\right.$ as $\left.B^{\prime} \neq \emptyset\right)$. 
Assume the claim is not true. Then $A^{\prime}-\{v\} \neq \emptyset$. Let $s \in A^{\prime}-\{v\}$. By definition, $\varphi_{L, \min }(s)<\varphi_{L_{1}^{\prime}, \min }(s) \leq \varphi_{L_{1}^{\prime}, \min }(v)$. Let $e=u v$. Then

$$
\begin{aligned}
\varphi_{L_{1}, \min }(u) & =\varphi_{L, \min }(u)+L_{\max }(e)-L_{\min }(e) \\
\varphi_{L_{1}^{\prime}, \min }(v) & =\varphi_{L, \min }(v)+L_{\max }(e)-L_{\min }(e) .
\end{aligned}
$$

As $\varphi_{L, \min }(u)=\varphi_{L, \min }(v)$, we have $\varphi_{L_{1}, \min }(u)=\varphi_{L_{1}^{\prime}, \min }(v)$. Hence $\varphi_{L_{1}, \min }(s)=$ $\varphi_{L, \min }(s)<\varphi_{L_{1}, \min }(u)$. But $B^{\prime}=\{u\}$ implies that $\varphi_{L_{1}, \min }(u) \leq \varphi_{L_{1}, \min }(x)$ for all $x \in A-\{v\}$. This is a contradiction.

Let $w \neq u, v$ be a vertex such that for any $x \neq u, v, \varphi_{L, \max }(x) \leq$ $\varphi_{L, \max }(w)$. Without loss of generality, we assume that $w \in B$. Then in the graph $G_{1}=G-v, w$ is a local maximum vertex with respect to $L_{1}$. Let $f_{1}$ be the partial $L_{1}$-weighting of $G_{1}$ that max-weights $w$. Let $L_{2}$ be the (1,2)-total list assignment of $G_{2}=G_{1}-w$ induced by $L_{1}$ and $f_{1}$. Let $S_{2}=S_{1} \cup Q_{L_{1}, \max }(w)$ and $T_{2}=T_{1}-N_{G_{1}}[w]$. Then $T_{2}=\emptyset$ and $S_{2} \subseteq\{u\} \cup(A-\{v\})$.

Now we shall prove that $V_{L_{2}, \min }\left(G_{2}\right)=\{u\}$. I.e., for any $z \in(A-\{v\}) \cup$ $(B-\{u, w\})$,

$$
\varphi_{L_{2}, \min }(u)<\varphi_{L_{2}, \min }(z) .
$$

If $z \in B-\{u, w\}$, then since $B^{\prime}=\{u\}$, it follows that

$$
\varphi_{L_{2}, \min }(z)=\varphi_{L_{1}, \min }(z)>\varphi_{L_{1}, \min }(u)=\varphi_{L_{2}, \min }(u) .
$$

If $z \in A-\{v\}$, then

$$
\varphi_{L_{2}, \min }(z)>\varphi_{L_{1}, \min }(z) \geq \varphi_{L_{1}, \min }(u)=\varphi_{L_{2}, \min }(u) .
$$

Let $f_{2}$ be the partial $L_{2}$-weighting of $G_{2}$ that min-weights $u$, and let $L_{3}$ be the (1,2)-total list assignment of $G_{3}=G_{2}-u$ induced by $L_{2}$ and $f_{2}$. Let $S_{3}=S_{2}-N_{G_{2}}[u]$ and $T_{3}=T_{2} \cup Q_{L_{2}, \min }(u)$. Then $S_{3}=T_{3}=\emptyset$. Since $G \neq K_{2,3}$, hence $G_{3} \neq K_{2}$. Hence $G_{3}$ has a proper $\left(L_{3} ; S_{3}, T_{3}\right)$-weighting. By repeatedly applying Theorem 2.3, we conclude that $G_{2}$ has a proper ( $\left.L_{2} ; S_{2}, T_{2}\right)$-weighting, and hence $G_{1}$ has a proper $\left(L_{1} ; S_{1}, T_{1}\right)$-weighting, and hence $G$ has a proper $\left(L ; S_{0}, T_{0}\right)$-weighting.

It was shown in [7] that complete bipartite graphs other than $K_{2}$ are 3 -edge weight choosable. By assigning every vertex weight 0 , we have the following corollary. 
Corollary 3.3. Every complete bipartite graphs other than $K_{2}$ are 2-edge weight choosable.

Corollary 3.3 can be stated as a result about matrices, which seems interesting.

Corollary 3.4. If $n+m \geq 3$, and $\mathcal{L}=\left[L_{i j}\right]$ is an $n \times m$ matrix where each $L_{i j}$ is a set of two real numbers, then there is an $n \times m$ real matrix $A=\left[a_{i j}\right]$ such that $a_{i j} \in L_{i j}$ and no row sum of $A$ is equal to a column sum of $A$.

Note that the induction proof of Theorem 3.2 cannot be used to give a direct proof of Corollary 3.3. This shows that even if our interests are in edge weight choosability of graphs, it can be helpful by considering the more general total weight choosability of graphs.

\section{Algebraic method}

Assume $G=(V, E)$ is a simple graph, where $V=\left\{v_{1}, v_{2}, \ldots, v_{n}\right\}$ and $E=$ $\left\{e_{1}, e_{2}, \ldots, e_{m}\right\}$. Denote by $X$ the set of variables $x_{v_{1}}, x_{v_{2}}, \ldots, x_{v_{n}}, x_{e_{1}}, x_{e_{2}}, \ldots, x_{e_{m}}$. For each vertex $v$ of $G$, let $Q_{v}=\sum_{e \in E(v)} x_{e}+x_{v}$. Fix an arbitrary orientation $\vec{G}$ of $G$. Let

$$
\tilde{P}(\vec{G})=\tilde{P}\left(x_{v_{1}}, x_{v_{2}}, \ldots, x_{v_{n}}, x_{e_{1}}, x_{e_{2}}, \ldots, x_{e_{m}}\right)=\prod_{u v \in E(\vec{G})}\left(Q_{v}-Q_{u}\right) .
$$

For each edge $u v$ (oriented from $u$ to $v$ ), $Q_{v}-Q_{u}$ is a polynomial of degree 1 with variable set $X$. So $\tilde{P}(\vec{G})$ is a polynomial of degree $m=|E(G)|$ with variable set $X$. For different orientations $\vec{G}$ of $G$, the polynomials defined may differ by a sign, which is irrelevant for our purpose.

Suppose $f$ is a total weighting of $G$. By definition, for each vertex $u$,

$$
\varphi_{f}(u)=Q_{u}\left(f\left(v_{1}\right), f\left(v_{2}\right), \ldots, f\left(v_{n}\right), f\left(e_{1}\right), f\left(e_{2}\right), \ldots, f\left(e_{m}\right)\right)
$$

is equal to the evaluation of the polynomial $Q_{u}$ with $x_{v}=f(v)$ for each $v \in V$ and $x_{e}=f(e)$ for each edge $e \in E$. Therefore $f$ is a proper total weighting of $G$ if and only if

$$
\tilde{P}\left(f\left(v_{1}\right), f\left(v_{2}\right), \ldots, f\left(v_{n}\right), f\left(e_{1}\right), f\left(e_{2}\right), \ldots, f\left(e_{m}\right)\right) \neq 0 .
$$

A mapping $\eta: V \cup E \rightarrow\{0,1, \ldots\}$ is called a valid index function if $\sum_{y \in V \cup E} \eta(y)=m$. For a valid index function $\eta$, let $c_{\eta}$ be the coefficient of 
the monomial $\prod_{y \in V \cup E} x_{y}^{\eta(y)}$ in the expansion of $\tilde{P}(\vec{G})$. It follows from the Combinatorial Nullstellensatz $[6,5]$ that if $c_{\eta} \neq 0$, and $|L(y)| \geq \eta(y)+1$ for all $y \in V \cup E$, then there exist $f(y) \in L(y)$ for $y \in V \cup E$ such that

$$
\tilde{P}\left(f\left(v_{1}\right), f\left(v_{2}\right), \ldots, f\left(v_{n}\right), f\left(e_{1}\right), f\left(e_{2}\right), \ldots, f\left(e_{m}\right)\right) \neq 0 .
$$

I.e., $G$ has a proper $L$-total weighting.

The coefficient $c_{\eta}$ of the monomial $\prod_{y \in V \cup E} x_{y}^{\eta(y)}$ is associated to the permanent of some matrices. Let $A_{\vec{G}}$ be the $m \times(n+m)$ matrix, with rows indexed by the edges of $\vec{G}$ and columns indexed by vertices and edges, defined as follows: If $e=u v$ is an edge of $\vec{G}$ oriented from $u$ to $v$, then let

$$
a_{e y}=\left\{\begin{array}{l}
1, \text { if } y=v \text { or } y \neq e \text { is an edge incident to } v \\
-1, \text { if } y=u \text { or } y \neq e \text { is an edge incident to } u \\
0, \text { otherwise. }
\end{array}\right.
$$

Then

$$
\tilde{P}(\vec{G})=\prod_{e \in E}\left(\sum_{y \in V \cup E} a_{e y} x_{y}\right) .
$$

For each $y \in V \cup E$, we denote by $A_{y}$ the column of $A_{\vec{G}}$ indexed by $y$. So

$$
A_{\vec{G}}=\left[A_{v_{1}}, A_{v_{2}}, \ldots, A_{v_{n}}, A_{e_{1}}, A_{e_{2}}, \ldots, A_{e_{m}}\right] .
$$

For a valid index function $\eta$, let $A_{\vec{G}}(\eta)$ be the $m \times m$ matrix in which the column $A_{y}$ occurs $\eta(y)$ times for each $y \in V \cup E$. It is known [4] that $c_{\eta} \neq 0$ if and only if the permanent of $A_{\vec{G}}(\eta)$ is non-zero, where the permanent of an $m \times m$ matrix $A=\left[a_{i j}\right]$ is defined as

$$
\operatorname{per}(A)=\sum_{\sigma} \prod_{j=1}^{m} a_{j \sigma(j)},
$$

here the summation is taken over all the permutations $\sigma$ of $\{1,2, \ldots, m\}$.

Thus by showing that certain matrices have non-zero permanent, we can prove results about total weight choosability of graphs. For any graph $G=(V, E)$, let $\eta_{G}^{*}$ be the valid index function defined as $\eta_{G}^{*}(e)=1$ for each edge $e$ and $\eta_{G}^{*}(v)=0$ for each vertex $v$. By the discussion above, if $A_{\vec{G}}\left(\eta_{G}^{*}\right)$ has non-zero permanent, then $G$ is $(1,2)$-choosable. It is not difficult to verify that for $G=K_{1,2}, K_{1,3}, K_{2,2}, K_{2,3}$, the permanent of $A_{\vec{G}}\left(\eta_{G}^{*}\right)$ is nonzero. Therefore these graphs are $(1,2)$-choosable. 
We believe that Conjectures 1.1 and 1.2 can be proved by using this method. To be precise, the following stronger conjectures are proposed in $[7,13]$ :

Conjecture 4.1. [13] For any graph $G$, there is a valid index function $\eta$ with $\eta(y) \leq 1$ for all $y \in V \cup E$ such that $\operatorname{per}\left(A_{\vec{G}}(\eta)\right) \neq 0$.

Conjecture 4.2. [7] For any graph $G$ without isolated edges, there is a valid index function $\eta$ with $\eta(v)=0$ for all $v \in V$ and $\eta(e) \leq 2$ for all $e \in E$ such that $\operatorname{per}\left(A_{\vec{G}}(\eta)\right) \neq 0$.

In [13], we had limited success and proved that if $G=K_{2, n}$, then $G$ is $(1,2)$-choosable, and if $G$ is a complete graph, or $G=K_{3, n}$, or $G$ is a tree or a generalized theta graph, then Conjecture 4.1 holds for $G$, and hence $G$ is $(2,2)$-choosable. We were unable to prove that general complete bipartite graphs are $(2,2)$-choosable by this method in [13]. In this paper, by using the max-min weighting method, we are able to prove a stronger result: if $G$ is a complete bipartite graph, then $G$ is $(1,2)$-choosable. The main result in [13] that complete graphs are $(2,2)$-choosable is also implied by Theorem 3.1. In this sense, the max-min method has been more successful. However, it seems to us that the hope of confirming Conjectures 1.1 and 1.2 is more likely to lie on the algebraic method. For this purpose, we would still like to see a proof of the results in this paper by the algebraic method. Because complete bipartite graphs other than $K_{2}$ are $(1,2)$-choosability, one naturally asks whether $\operatorname{per}\left(A_{\vec{G}}\left(\eta_{G}^{*}\right)\right) \neq 0$ for all complete bipartite graphs $G \neq K_{2}$. But this question has a negative answer.

Theorem 4.3. If $G$ is a bipartite graph with an odd number of edges and there is an automorphism of $G$ that interchanges the two partite sets, then $\operatorname{per}\left(A_{\vec{G}}\left(\eta_{G}^{*}\right)\right)=0$. In particular, if $n$ is odd and $G=K_{n, n}$, then $\operatorname{per}\left(A_{\vec{G}}\left(\eta_{G}^{*}\right)\right)=$ 0 .

Proof. The permanent of the matrix $A_{\vec{G}}\left(\eta_{G}^{*}\right)$ depends on the orientation of the edges. Changing the orientation of one edge will change the sign of the permanent. Assume the two partite sets of $G$ are $A$ and $B$. Let $\vec{G}_{1}$ be the orientation of $G$ in such a way that all the edges are from $B$ to $A$. Let $\vec{G}_{2}$ be the orientation of $G$ in which all the edges are oriented from $A$ to $B$. Since $\vec{G}_{2}$ is obtained from $\vec{G}_{1}$ by changing the orientation of an odd number of edges, their permanents differ by a sign. However, by our assumption, $\vec{G}_{2}$ 
and $\vec{G}_{1}$ are isomorphic, so they have the same permaments. Therefore their common permanent is 0 .

This result shows that not every result on total weight choosability of graphs can be proved by the method of calculating the permanents of some matrices. Nevertheless, it has not yet crushed our faith on Conjectures 4.1 and 4.2. For complete bipartite graphs, based on some experiments, we suspect that if $n \neq m$ or $n=m$ are even, then for $G=K_{n, m}, A_{\vec{G}}\left(\eta_{G}^{*}\right)$ has nonzero permanent. We can prove this for some special cases.

Theorem 4.4. If $n, q$ are positive integers such that $n$ has a prime factor $p>q$, then for $G=K_{n, q}, A_{\vec{G}}\left(\eta_{G}^{*}\right)$ has nonzero permanent.

Proof. As the implication of this theorem to total choosability of graphs is already proved, we shall just sketch an outline of the proof and omit some details.

Assume the two partite sets of $G$ are $A=\left\{v_{1}, v_{2}, \cdots v_{n}\right\}$ and $B=$ $\left\{u_{1}, u_{2}, \cdots u_{q}\right\}$. We orient the edges of $G$ so that all the edges are oriented from $B$ to $A$. We shall prove that $\operatorname{per}\left(A_{\vec{G}}\left(\eta_{G}^{*}\right)\right)$ is not a multiple of $p$, and hence $\operatorname{per}\left(A_{\vec{G}}\left(\eta_{G}^{*}\right)\right) \neq 0$.

Suppose $e=u v$ is an edge of $G$ oriented from $u$ to $v$, then the column $A_{e}$ can be expressed as $A_{e}=A_{v}+A_{u}$. It is known (and easy to see) that the following is true: Assume $A$ is a matrix, and $A_{i}$ is a column of $A$, and $A_{i}=A_{i}^{\prime}+A_{i}^{\prime \prime}$. Let $A^{\prime}$ be obtained from $A$ by replacing $A_{i}$ with $A_{i}^{\prime}$ and $A^{\prime \prime}$ be obtained from $A$ by replacing $A_{i}$ by $A_{i}^{\prime \prime}$. Then

$$
\operatorname{per}(A)=\operatorname{per}\left(A^{\prime}\right)+\operatorname{per}\left(A^{\prime \prime}\right)
$$

The following observation can be derived from the definition of permanent (cf. [4])

Observation 4.5. If $M$ is an integer matrix, and $k$ of the columns of $M$ are identical, then $\operatorname{per}(M)$ is a multiple of $k !$.

Suppose $F$ is a subset of $E$ and $F^{\prime}$ is a subset of $F$. Let $A_{F^{\prime}, F}$ be the matrix obtained from $A_{\vec{G}}\left(\eta_{G}^{*}\right)$ by replacing, for each $e=u v \in F^{\prime}$, the column $A_{e}$ by $A_{u}$, and replacing for each $e=u v \in F-F^{\prime}$, the column $A_{e}$ by $A_{v}$. For any subset $F$ of $E$, by repeatedly applying (1), we have

$$
\operatorname{per}\left(A_{\vec{G}}\left(\eta_{G}^{*}\right)\right)=\sum_{F^{\prime} \subseteq F} \operatorname{per}\left(A_{F^{\prime}, F}\right)
$$


Let $F_{1}=\left\{u_{1} v_{j}: j=1,2, \ldots, n\right\}$. For $k=0,1, \ldots, n$, let

$$
\mathcal{F}_{1, k}=\left\{F^{\prime} \subseteq F_{1}:\left|F^{\prime}\right|=k\right\}
$$

It is easy to see that if $F^{\prime}, F^{\prime \prime} \in \mathcal{F}_{1, k}$, then

$$
\operatorname{per}\left(A_{F^{\prime}, F_{1}}\right)=\operatorname{per}\left(A_{F^{\prime \prime}, F_{1}}\right) \text {. }
$$

If $\left|F^{\prime}\right|=k$ and $k \geq p$, then by Observation $4.5,\left|\operatorname{per}\left(A_{F^{\prime}, F_{1}}\right)\right|$ is a multiple of $k$ !, which is a multiple of $p$.

If $1 \leq k<p$, then $\left|\mathcal{F}_{1, k}\right|=\left(\begin{array}{l}n \\ k\end{array}\right)$ is a multiple of $p$. Hence the sum $\sum_{F^{\prime} \in \mathcal{F}_{1, k}} \operatorname{per}\left(A_{F^{\prime}, F_{1}}\right)$ is a multiple of $p$. Therefore

$$
\operatorname{per}\left(A_{\vec{G}}\left(\eta_{G}^{*}\right)\right) \cong \operatorname{per}\left(A_{\emptyset, F_{1}}\right) \quad(\bmod p) .
$$

Let $F_{2}=\left\{u_{j} v_{s}: s=1,2, \ldots, n, j=1,2\right\}$. Then

$$
\operatorname{per}\left(A_{\emptyset, F_{1}}\right)=\sum_{F^{\prime} \subseteq F_{2}, F^{\prime} \cap F_{1}=\emptyset} \operatorname{per}\left(A_{F^{\prime}, F_{2}}\right) .
$$

Repeat the above process, we can prove that

$$
\operatorname{per}\left(A_{\emptyset, F_{1}}\right) \cong \operatorname{per}\left(A_{\emptyset, F_{2}}\right) \quad(\bmod p) .
$$

For $i \geq 2$, let $F_{i}=\left\{u_{j} v_{s}: s=1,2, \ldots, n, j=1,2, \ldots, i\right\}$. By repeating the process above, we can prove that

$$
\operatorname{per}\left(A_{\vec{G}}\left(\eta_{G}^{*}\right)\right) \cong \operatorname{per}\left(A_{\emptyset, F_{1}}\right) \cong \cdots \cong \operatorname{per}\left(A_{\emptyset, F_{i}}\right) \quad(\bmod p)
$$

Therefore

$$
\operatorname{per}\left(A_{\vec{G}}\left(\eta_{G}^{*}\right)\right) \cong \operatorname{per}\left(A_{\emptyset, E}\right) \quad(\bmod p) .
$$

But it is easy to verify that $\operatorname{per}\left(A_{\emptyset, E}\right)=(q !)^{n} \nsucceq 0(\bmod p)$.

It would be interesting to prove that for any complete bipartite graph $G=K_{n, m}$, unless $n=m$ is odd, we have $\operatorname{per}\left(A_{\vec{G}}\left(\eta_{G}^{*}\right)\right) \neq 0$. If this is true, then it follows from a result in [13] that Conjecture 4.1 holds for all complete bipartite graphs. 


\section{References}

[1] L. Addario-Berry, R.E.L.Aldred, K. Dalal, B.A. Reed, Vertex colouring edge partitions, J. Combin. Theory Ser. B 94 (2005), 237-244.

[2] L. Addario-Berry, K. Dalal, B.A. Reed, Degree constrianed subgraphs, Proceedings of GRACO2005, Volume 19, Electron. Notes Discrete Math., Amsterdam (2005), 257-263, Elsevier.

[3] L. Addario-Berry, K. Dalal, C. McDiarmid, B.A. Reed, A. Thomason, Vertex-colouring edge-weightings, Combinatorica 27 (2007), 1-12.

[4] N. Alon and M. Tarsi, A nowhere zero point in linear mappings, Combinatorica 9 (1989), 393-395.

[5] N. Alon and M. Tarsi, Colorings and orientations of graphs, Combinatorica, 12 (1992), 125-134.

[6] N. Alon and M. Tarsi, Combinatorial Nullstellensatz, Combin. Prob. Comput. 8 (1999), 7-29.

[7] T. Bartnicki, J. Grytczuk and S. Niwczyk, Weight choosability of graphs, preprint, 2007.

[8] G. Chang, C. Lu, J, Wu and Q. Yu, Vertex coloring 2-edge weighting of bipartite graphs, preprint, 2007.

[9] M. Karoński, T. Łuczak, A. Thomason, Edge weights and vertex colour, J. Combin. Theory Ser. B 91(2004), 151-157.

[10] M. Kalkowski, M. Karonski and F. Pfender, Vertex-coloring edgeweightings: towards the 1-2-3-Conjecture, manuscript, 2008.

[11] J. Przybylo and M. Wozniak, 1,2-conjecture, preprint, 2007.

[12] J. Przybylo and M. Wozniak, 1,2-conjecture II, preprint, 2007.

[13] T. Wong and X. Zhu, Total weight choosability of graphs, preprint, 2008. 